\title{
HOW DO FAKE NEWS PROPAGATORS EXPLOIT SOCIAL ALGORITHMS TO PROMOTE THEIR CONTENTS?
}

\author{
Ittipon Rassameeroj ${ }^{1}$ and Shyhtsun Felix $\mathrm{Wu}^{2}$ \\ ${ }^{1}$ Faculty of ICT, Mahidol University, Nakhon Pathom, Thailand \\ ${ }^{2}$ Department of Computer Science, UC Davis, Davis, CA, USA
}

\begin{abstract}
Social media has currently become a primary platform to consume and exchange information. Instead of browsing through media source websites we are interested, we have enabled social media to deliver related contents to us. As a black box, social algorithms were designed and trained to pick up, filter, and rank the most relevant and desired contents to be delivered to each individual one of us. In addition, social media allows us to add more contents as a comment on an original post, which we call user-created content. Unfortunately, a lot of fake news contents have been created and disseminated in Facebook fan pages. Many of them could draw many attentions from users. Our research question is how do fake news propagators successfully make their contents be more visible and disseminate it to many users through social algorithms? In this work, we explored behavior and activities of fake news propagators and their trapped recipient to understand how social algorithms widely delivered those contents to many users on Facebook fan pages. From our SINCERE data, we extracted top 300 fake news comments that got the highest number of participants as our data set. We statistically analyzed the data set based on our hypotheses, which were mainly from our previous work of reverse-engineering for content delivery algorithms in social media and from what we observed from the SINCERE data. Our main contribution for this work is to understand behaviors, activities, and strategies of fake news propagators who successfully disseminated their contents to many users through social algorithms on Facebook.
\end{abstract}

\section{KEYWORDS}

Fake News, Misinformation, Social Algorithms, Social Media, Facebook Fan Pages, Online Public Communities

\section{INTRODUCTION}

In past decade, online content consumption behavior has been changed. We had to browse through websites of media source that we are interested or use online search engine tools to search and bring us to the online content we desired. On the contrary, social media has currently become a primary platform to consume and exchange information. We have enabled social media to deliver online contents to us. In addition, social media platforms allow us to add contents by creating comments on an original content, which we call user-created content. For Facebook, many users not only focus on the original posts, but also consume other user comments nowadays. Like online search engine tools, social media systems have some mechanisms to pick up, filter, and rank the most relevant and desired contents, included both original posts and user comments, to be delivered to each individual one of us, which we call social algorithms. Conceptually, social algorithms are usually designed and trained by usually using social network data, personalization, and online activities. However, as a black box, we do not really know how social algorithms work. It is obvious that even we have more and more online contents, but we cannot control and customize our content delivery algorithms much in social media systems.

A user-created content (or a user comment) could be opinion, more related information, other aspects of information, or even misinformation. Unfortunately, a lot of misinformation contents, like fake news, have been created and disseminated in social media. Many of them could successfully draw attentions from many users more than even other good contents, such as fact-checking contents. For example, on Facebook, those fake news contents got many likes or reactions. Initially, our simple question is many users were interested in those contents, or social algorithms made those contents to be more visible to them? We strongly believe that social algorithms play an important role in that. If so, our main research question is how do fake news propagators make use of social algorithms to make their contents to be more visible to many users? 
In this work, we explored social algorithms for fake news contents in Facebook fan pages and public communities. Also, we tried to find main factors or behavioral patterns that fake news propagators made their fake news contents to be more visible and be delivered to many other users via social algorithms. We empirically defined hypotheses, which mainly were from what we have observed from our SINCERE data and from our previous work of reverse-engineering for content delivery algorithms in social media, based on behaviors and activities of fake news propagators. We statistically analyzed our data set based on our hypotheses to find main factors that fake news contents could be widely delivered to many users though social algorithms on Facebook fan pages.

The remainder of this paper is organized as follows. We motivated our study with additional related work and backgrounds in Section 2. In Section 3, we briefed our conceptual approach. In Section 4, we introduced our SINCERE data and the data set we used in this work. Also, we showed top 5 fake news URLs we found in the SINCERE data and top 10 Facebook fan pages that had the largest number of fake news comments. In Section 5, we presented our hypotheses and experimental results of data analysis. Finally, in we discussed our limitations for this work, and we summarized this paper in Section 6.

\section{RELATED WORKS}

Lazer (2015) presented some ideas, overview, and brief definition of social algorithms. The author also gave some examples to show how social algorithm had an impact on choosing and ranking all content on the Internet for users. On the other hand, Yang (2017) presented social algorithms in terms of computational algorithms, which is a special class of algorithms for solving optimization problems. The author showed the algorithms that did not focus on only social media systems, but they could be applied for general context, mainly nature-inspired, population-based algorithm for optimization.

Social media systems, like Facebook and Twitter, have become one of the main channels to diffuse information rapidly. Basic techniques that have been used for their content delivery algorithms include personalization, interests, and online activities. In addition, the strength of weak ties theory (Granovetter, 1973 ) is one of the most popular techniques applied to content delivery algorithm in most of social media providers. Gilbert and Karahalios (2009) presented a predictive model that maps social media data to tie strength in online social networks. For example, contents that were created from friends whom we frequently interacted with should be picked and shown first. Bakshy (2012) presented how strong and weak ties had influences in terms of information propagation in social networks.

A very challenging research question is how can we evaluate that contents filtered by social algorithms are the most desired we would like to see? There are existing researches investigating bias in social algorithms. Pariser (2011) presented filter bubble, which personalization made people see different viewpoints of content. In addition, since we all do not really know how social algorithms work, there are some concerns about bias of algorithmically selected content. Furthermore, ideological diverse issue is another concern for social algorithms. Flaxman, Goel, and Rao (2016) pointed out that both search engines and social media systems may use ideological distance as a factor to pick a content up and push it to users. Also, those systems are associated with an increase in the mean ideological distance between individuals.

We did reverse engineering of social algorithms on Facebook fan page. They have focused on top posts that had a largest number of comments in news media pages (Rassameeroj and $\mathrm{Wu}, 2019$ ). However, they did not investigate misinformation contents and did not analyze behaviors and activities of comment authors.

Del Vicario et al (2016) did governing misinformation spreading through a thorough quantitative analysis and focused on how Facebook users consume information related to two distinct narratives: scientific and conspiracy news. In (Shao et al, 2017), the authors analyzed 14 million messages spreading 400 thousand claims on Twitter during and following the 2016 U.S. presidential campaign and election. They found that accounts that actively spread misinformation are significantly more likely to be bots. In (Del Vicario et al, 2019), they analyzed Italian Facebook dataset to identify topics that are susceptible to misinformation. Vosoughi, Roy, and Aral (2018) investigated the differential diffusion all of the verified true and false news stories distributed on Twitter from 2006 to 2017. They found that false news was more novel than true news, which suggested that people were more likely to share novel information. Hu et al (2013) did modeling the messages and pathway propagation in a social network based on social network users and structures. 


\section{CONCEPTUAL APPROACH}

Basically, our goal is to explore how fake news propagators exploit social algorithms to make their contents be more visible to many users based on their behavior and activities. In this work, we considered user comments, which contained fake news URLs or domains, in posts in Facebook fan pages and communities, as fake news contents. We focused on Facebook fan pages because they are popular targets for fake news. Since all users can interact with anyone as long as they are members in the same communities regardless of relationship, it is very easy to disseminate malicious contents on there. In order to work towards that, we analyzed behaviors or activities of fake news propagators and their recipients based on our hypotheses. We assume that users, who liked, reacted, or replied to the fake news comments, are the recipients. Our hypotheses empirically were from our previous work (Rassameeroj and $\mathrm{Wu}, 2019$ ), what we have noticed in Facebook features, and what we observed in our data set. From the data set, we statistically analyzed each fake news comment in each original post independently.

\section{DATA}

We used our Social Interactive Networking and Conversation Entropy Ranking Engine (SINCERE) data (Erlandsson et al, 2015). It was crawled via Facebook API from public interaction data on almost all Facebook fan pages and public communities from 2008 to early 2018. The SINCERE data is composed of almost all posts, comments, likes, reactions (love, haha, wow, anger, sad), and shares. Table 1 presents total amount of the SINCERE data we have.

Table 1. Total amount of data on SINCERE

\begin{tabular}{lr}
\hline Data type & Amount of data \\
\hline Facebook fan pages & $4,706,324$ \\
Posts & $342,998,071$ \\
Comments & $6,432,081,954$ \\
Likes and reactions & $132,876,481,459$ \\
Replies & $1,110,940,833$ \\
Shares & $163,301,864$ \\
\hline
\end{tabular}

To get all fake news contents (comments) from the SINCERE data, we firstly used a list of fake news URLs and domains mainly collected from Shao et al (2017) and fact-checking websites, such as snopes.com, factcheck.org, politifact.com, washingtonpost.com, mediabiasfactcheck.com, and truthorfiction.com. From the SINCERE data, Table 2 shows some samples of our fake news URLs or domains, which is the top five of fake news URLs that were attached in comments in Facebook fan pages.

Table 2. Top five Fake News URLs or domains attached in comments in Facebook fan pages

\begin{tabular}{cccc}
\hline No. & Fake news URLs/domains & The number of comments contained the URL & The number of fan pages \\
\hline 1 & infowars.com & 73,610 & 4,061 \\
2 & thegatewaypundit.com & 59,724 & 2,970 \\
3 & globalresearch.ca & 41,632 & 3,593 \\
4 & naturalnews.com & 37,934 & 3,892 \\
5 & beforeitsnews.com & 27,459 & 2,644 \\
\hline
\end{tabular}

From the SINCERE data we have in Table 1, we extracted top 300 comments contained fake news URLs, which got the highest number of reactions from users, to be our data set. In addition, the total number of fake news comments on our SINCERE data is 533,973 comments. The total number of reactions of those fake news comments is 361,758 , which we could imply that is the number of infected users from fake news contents.

As we analyzed behavior of both fake news propagators and their participants, we would like to make sure that their participants naturally received the fake news contents from social algorithms only, not from other techniques of fake news propagators, such as likebait. Thus, we investigated whether all fake news comments in our data set got reactions from normal users, not from bot accounts or users who only 
participated in those fake news comments for some other reasons. For example, if most participants of a fake news comments did not participate in other comments, or they did not have other activities, it is most likely that fake news comment might be promoted by using fake news propagators' techniques (such as generating likebait from bot accounts), not by social algorithms.

Hence, for each of top 300 fake news comments from our data set, we would like to see how many participants reacted to other comments. Figure 1 shows percentage of participants who reacted to other comments (on y-axis) for each of top 300 fake news comments (on x-axis). Please ignore comments number 62 and 184 in the figure because we have lost some data for these comments. In the figure, we could imply that all fake news comments from our data set did not generate from any bot accounts because most of participants also interacted with other comments as usual, not only with the fake news comments.

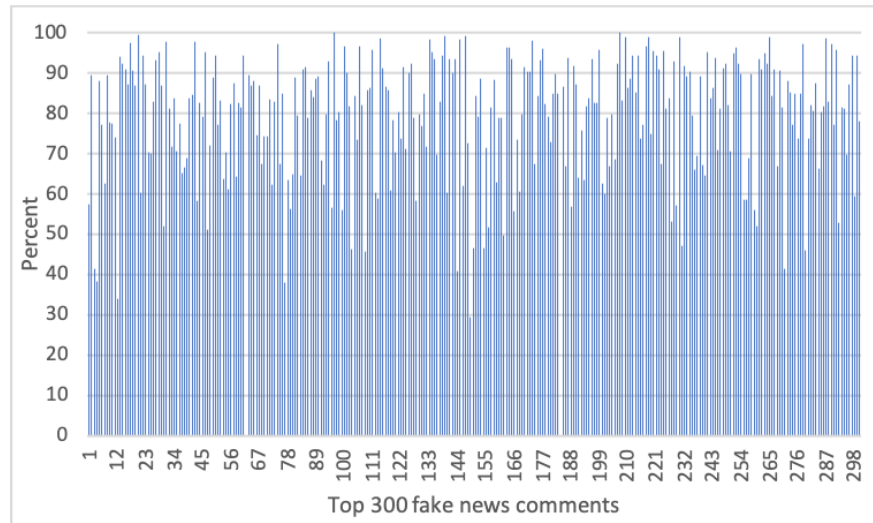

Figure 1. Percentage of reaction users who reacted to other comments for each of top 300 fake news comments

Before we investigated fake news propagators behavior, we would like to present top 10 targets of Facebook fan pages that have the largest number of fake news contents from the whole SINCERE data in Table 3. We also show the number of reactions for those comments in the last column in the table, which we simply imply the total number of users who might be trapped by a fake news.

Table 3. Top 10 Facebook fan pages with the largest number of fake news comments

\begin{tabular}{cccc}
\hline No. & Facebook fan pages & The number of fake news URL comments & The number of reactions \\
\hline 1 & Fox News & 40,811 & 19,069 \\
2 & Hillary Clinton & 26,281 & 6,649 \\
3 & The White House & 21,232 & 7,046 \\
4 & Barack Obama & 20,017 & 7,004 \\
5 & Donald J. Trump & 15,490 & 5,279 \\
6 & MSNBC & 12,031 & 4,661 \\
7 & CNN & 11,529 & 6,307 \\
8 & Alex Jones & 6,649 & 19,455 \\
9 & Ted Cruz & 5,645 & 1,883 \\
10 & RT & 5,472 & 8,015
\end{tabular}

\section{HYPOTHESES AND EXPERIMENTS}

In this section, we present hypotheses of fake news propagator strategies and behavior. Then, we present experimental results from statistically analyze the data set based on interaction, activities, and behavior of fake news propagators (Section 5.1 and 5.2) and their recipients (Section 5.3).

\subsection{Creation Time}

Our first hypothesis is that if a fake news comment is created very early right after an original post is published, social algorithms will probably choose that comment to be the top comment for the posts, which 
means most users will see that comment first. Hence, we analyze the data set to see how early fake news comments were created after their original posts, and the result is presented in Figure 2. From the result, we have found that many fake news comments were created very early. $26 \%$ of them were created within two minutes after their posts, $19 \%$ and $17 \%$ of them were created in first five and 10 minutes after the posts respectively. It is obvious fake news content creator use this strategy to try to get a lot of attention from users.

In addition to the times when the fake news contents were created, we are also interested in their position or chronological order in that post. Even we have just shown the comments, that were created very early after the posts, were apparently seen by many users, we believe those comments might not be the first comment in their posts. From (Rassameeroj and Wu, 2019), the authors shown that users who create comments earlier in a post will very likely see other comments created later because of Facebook notification features. For this reason, our next hypothesis is a fake news comment might be created after a few of other comments were created in the same post. Thus, users who made comments before the fake news comment may see it, and they very likely reacted, such as liking, to it if they were still online, so the fake news comment seems credible because of getting a few likes early.

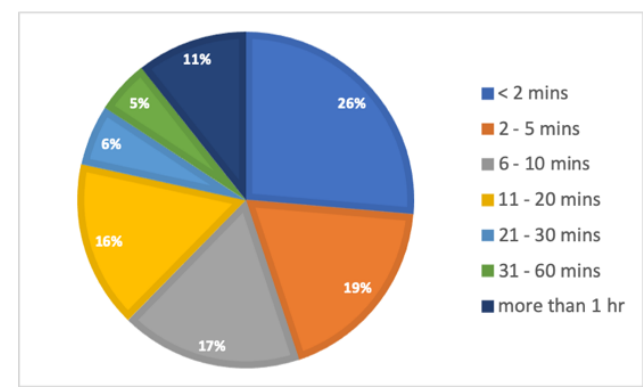

Figure 2. How early top fake news comments had been created after original posts were created

Figure 3 presents chronological order of the top 150 fake news comments (on x-axis) from the data set. Since we have found their orders very vary, we simply scale them as 0 to 100 in Figure 3 (on y-axis), which 0 represents the first comment while 100 represents the last comment in a post. From the result, most of top fake news comments were not created as the first comment in their post.

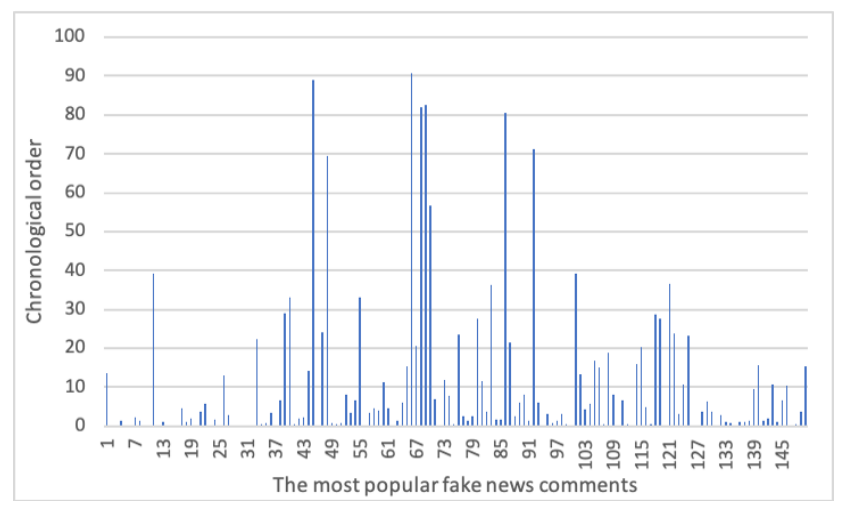

Figure 3. Chronological order of top 150 fake news comments in its post

\subsection{Interaction Behavior in a Post}

In this section, we tried to see interaction behavior of fake news propagators from our data set. Our hypothesis is that they might have some interactions to make social algorithms to understand that they are very active users so that their contents will be promoted to be more visible. We have initially listed all possible interaction behavior then have analyzed our data set to see their activities pattern. 


\subsubsection{Did they Like or React the Posts?}

Rassameeroj and $\mathrm{Wu}$ (2019) has been stated that if two users react to a post, and one of them makes a comment, then another user probably see that comment. We believe that reaction a post may be a factor to promote the content. From the data set, there are 93 of 300 fake news authors $(31 \%)$. Thus, based on our result and (Rassameeroj and $\mathrm{Wu}, 2019)$, reacting a post might be a good factor to promote contents.

\subsubsection{Did they make other Comments before and after their Fake News Comment in the Post?}

There are 25 of 300 fake news propagators (8.33\%) created other comments before their fake news comments. The average number of comments they created is 1.44 comments. Also, 64 of them (21.33\%) made more comments after the fake news comments, and the average number of comments they created is 2 comments per author. Hence, creating more comments might not be a good strategy to exploit social algorithms.

\subsubsection{Did they Click Like or Reaction on other Comments?}

93 of 300 fake news authors (31\%) liked or reacted other comments that were made before their fake news contents, and the average number of comments they reacted is 28 . However, 180 of 300 authors (60\%) liked or reacted other comments that were created after their fake news comments, and the average number of comments they reacted is 22 . That is their obvious behaviors, and it might be a strategy to promote their fake news contents be more visible to many users.

As a result, we have analyzed the data set more to see how they make use of social algorithms in that case. For each of fake news comments, we have investigated the reactions they got to see how many of them were from users who got liked or reacted from the fake news propagator. For example, suppose user $a$ and $b$ create comments in a post, and user $x$ likes comments of user $a$ and $b$. Then, user $x$ creates a fake news comment. We would like to see whether $x$ will get likes from $a$ and $b$. From the result, unfortunately, most of fake news comments got small reactions from users who got reactions from fake news authors. The average percentage of reactions from users who got likes from the fake news authors is $1.04 \%$, which is very small. As a result, recipients of fake news comments did not come from users who got reactions from fake news propagators, but the recipients might from users who did not make any comment. Accordingly, reactions to other comments may be still a good strategy to exploit social algorithms to cause users to see the their contents somehow based on the behavior of fake news propagators.

\subsubsection{Did they Reply to Other Comments?}

From the data set, 17 fake news propagators (5.67\%) replied to other comments made before their fake news comments, and the average number of replies is 1.7. In addition, 29 of 300 fake news propagators (9.67\%) replied to other comments that were created after their fake news comments, and the average number of replies for that is 1.9 . Consequently, we conclude that replying other comments is not a good way to promote contents.

\subsection{Exploring Reaction Users}

We have just done reverse engineering of how fake news propagators make use of social algorithms to promote the contents from their activities and interactions so far. In order to understand all aspects, in this section, we would like to shift from the author side to recipient side or users who saw reacted to those fake news contents. We believe if we see activities and behaviors of reaction users, we may correlate their patterns to understand where reactions were from. That is, we may understand why fake news comments were delivered to them. In this section, when we mention reaction users, that means users who liked, reacted, or replied to the fake news comments in the data set.

\subsubsection{Reactions from Users who Reacted to the Post}

We have found that many recipients, who reacted to the fake news comments, are from users who liked or reacted the original post of that comment. Figure 4 shows percentage of users, who reacted the post, from all reaction users of the top 150 fake news comment, which is on x-axis. That could imply that the top fake news comments were visible around the same time when users saw the post. 


\subsubsection{Reactions from Users who made Comments in the Post}

In (Rassameeroj and $\mathrm{Wu}, 2019$ ), the authors have presented a hypothesis saying that users who made a comment in a post may see other comments in the same post. Accordingly, we would like to see comment activities of users who had reactions to the fake news comments. Figure 5 presents percentage of reaction users who also made comments in the same post. Y-axis is represented the number of top fake news comments from 300 comments in the data set, and $\mathrm{x}$-axis is represented ranges of percentage of reaction users who made comment(s) in the same post. Most reaction users usually made $1-1.63$ comments per post in average.

\subsubsection{Did Reaction users also React to other Comments?}

From all reaction users of 300 fake news comments in the data set, Figure 6 presents the number of other comments that reaction users liked in each post. They mostly liked about $1-5$ comments in a post. From Figure 6, we could imply that if the number of likes is small, we might assume that social algorithms deliver a few contents with the fake news comments at that time. In addition, since the number of likes is quite small, most reaction users should not be like clicker users.

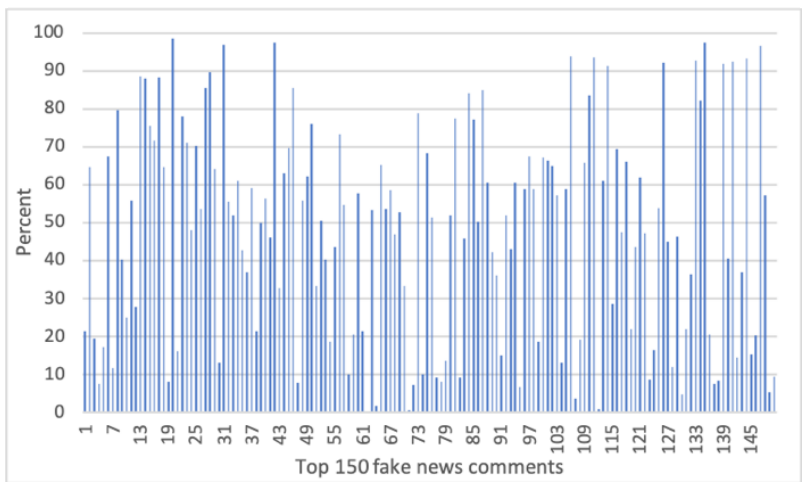

Figure 4. Percentage of users who participate in top 150 fake news comments and reacted to the post

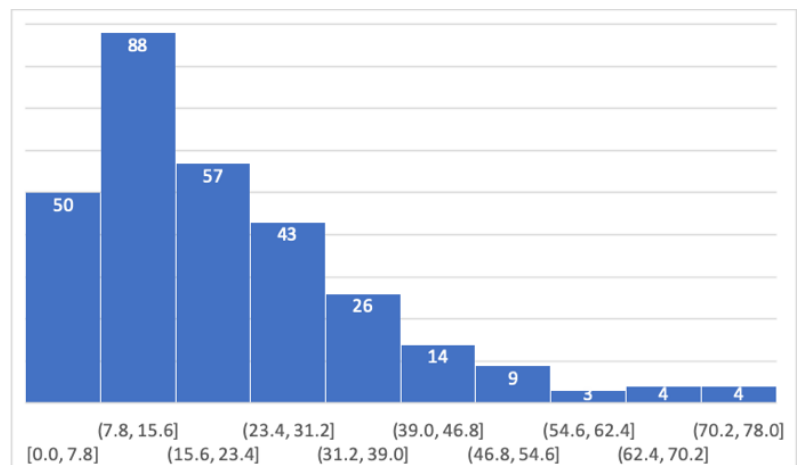

Figure 5. Percentage of fake news reaction users who made comments in the same post

\section{CONCLUSION}

We analyzed activities and behaviors of fake news propagators to understand how they make use of social algorithms to make their contents be more visible to many users. We expected to see some participation patterns of authors to promote their contents. One apparent factor to make their contents to be seen by many users is to post early after the original posts. Besides, we also analyzed behavior of users who reacted to all fake news comments to understand how reactions of the fake news contents were from. We figured out that reactions of fake news comments were from users who reacted the posts and comments the posts. 
Furthermore, we explored interaction and activities history of fake news propagators across different posts in the same page for one week and one month before. Unfortunately, we have not found any behavior that can be influence factors for them to promote the contents. Our main contribution for this work is to understand behaviors, activities, and strategies of fake news propagators who successfully disseminated their contents to many users through social algorithms on Facebook. We strongly believe our contribution could support other researches to understand fake news propagators in social media systems.

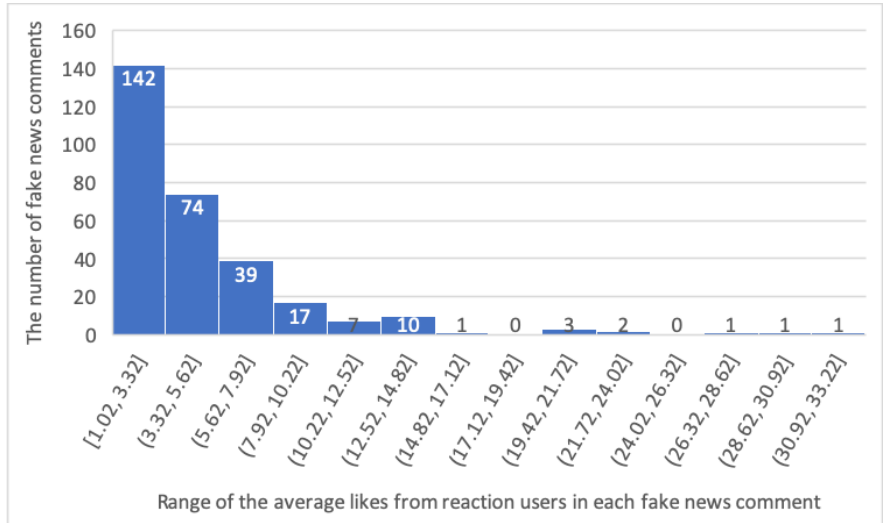

Figure 6. Percentage range of reaction users who reacted to other comments for each of 300 fake news comments

\section{REFERENCES}

Bakshy, E. et al, 2012. The role of social networks in information diffusion. Proceedings of The $21^{\text {st }}$ International Conference on World Wide Web. New York, NY, USA, pp. 519 - 528.

Del Vicario, M. et al, 2016. The spreading of misinformation online. Proceedings of the National Academy of Sciences, 113(3), pp. $554-559$.

Del Vicario, M. et al, 2019. Polarization and fake news: Early warning of potential misinformation targets. ACM Transactions on the Web, 13(2).

Erlandsson, F. et al, 2015. Crawling online social networks. Proceedings of $2^{\text {nd }}$ European Network Intelligence Conference, pp. $9-16$.

Flaxman, S., Goel, S., Rao, J.M., 2016. Filter bubbles, echo chambers, and online news consumption. Public Opinion Quarterly, 80(S1), pp. 298 - 320.

Gilbert, E., Karahalios, 2009. Predicting Tie Strength with Social Media. Proceedings of the SIGCHI Conference on Human Factors in Computing Systems, New York, NY, USA, pp. 211 - 220.

Granovetter, M.S., 1973. The Strength of Weak Ties. American Journal of Sociology, 78(6), pp. 1360 - 1380.

$\mathrm{Hu}, \mathrm{X}$. et al, 2013. Social Spammer Detection in Microblogging. Proceedings of IJCAI International Joint Conference on Artificial Intelligence, pp. $2633-2639$.

Lazer, D., 2015. The Rise of The Social Algorithm. Science, 348(6239), pp. 1090 - 1091.

Pariser, E., 2011. The Filter Bubble: What the Internet Is Hiding from You. Penguin Group.

Rassameeroj, I., Wu, S.F., 2019. Reverse Engineering of Content Delivery Algorithms for Social Media Systems. Proceedings of $20196^{\text {th }}$ International Conference on Social Network Analysis, Management, and Security, Granada, Spain, pp. $196-203$.

Shao, C. et al, 2017. A Platform for Tracking Online Misinformation. Association for Computing Machinery, pp. $745-750$.

Shao, C. et al, 2017. The Spread of Fake News by Social Bots, Nature Communication.

Vosoughi, S., Roy, D., Aral, S., 2018. The Spread of True and False News Online, Science, pp. 1146 - 1151.

Yang, X.S., 2017. Social Algorithms, Springer Berlin Heidelberg, pp. 1 - 15. 